\title{
Exploring which foods can be used as high protein carriers for adults older than 50 years
}

\author{
B. Curran and M. Dermiki \\ Faculty of Science, Department of Health and Nutritional Sciences, Institute of Technology Sligo, Ash Lane, F91 \\ YW50, Sligo, Ireland
}

The world population nowadays is ageing, which results in a shift towards higher numbers of people older than 65 years ${ }^{(1)}$. This increasing lifespan is not always matched with increased years spent in good health. Malnutrition, and particularly decreased protein intake is one of the leading causes of frailty among older adults ${ }^{(2)}$. Research has shown that protein enrichment of existing food products could be an effective way of enhancing protein intake ${ }^{(3)}$.

This study aimed to explore potential food products that could be used as high-protein carriers by surveying adults older than 50 years; addressing future older adults (50-64 years) and current older adults (older than 65 years). The developed questionnaire included questions related to sociodemographic characteristics (age, marital status, education, gender, financial status, place of residence), dietary and food purchasing habits, food choice motives and knowledge of protein function. Participants were also asked to state their willingness to consume 15 predetermined products as potential high-protein carriers ${ }^{(3)}$. Non-parametric tests were conducted $^{(4)}$ to investigate the effect of the participants' characteristics on the willingness to consume the products under investigation.

In total 61 participants completed the survey (57\% female). Most of them (68\%) belonged to the future older adults' group (50-64 years) and the majority lived in a town $(42 \%)$ or in a small village $(34 \%)$. A significant proportion $(40.7 \%)$ had little or no knowledge of protein function within the body and $74.6 \%$ were unaware of protein RDI.

While there was no effect of most of the sociodemographic characteristics on willingness to consume the food products under investigation, there was a gender effect on the willingness to consume yoghurt, as revealed from a Mann-Whitney $\mathrm{U}$ test $(\mathrm{U}=377, \mathrm{p}=$ $0.005)$ with female participants more willing to consume yoghurt (mean willingness score 3.26) than male (1.95). Friedman test $\left(\chi^{2}(14)=61.84, p<0.0001\right)$ on the willingness to consume the different products and post-hoc analysis using the Nemenyi's test, showed that spreads would be most preferred products, while meat substitutes and crisps would be the least preferred ones.

There were differences among the participants in the ranking of the food choice motives, as shown from a Friedman test $\left(\chi^{2}(7)\right.$ $=67.56, \mathrm{p}<0.0001)$. Nemenyi's test revealed that the health benefit, organoleptic properties, price and preparation were equally important food choice motives for the specific group, while environmental concern was the least important one.

From this pilot study it can be suggested that when exploring potential high-protein carriers, food producers should attempt to incorporate protein in products perceived as healthy, easy to prepare, without compromising their sensory properties. It may also be necessary to develop a variety of products to account for the heterogeneity of this age group. Finally, it is important to raise awareness in protein function.

1. WHO (2019) [Available online: https://www.who.int/news-room/fact-sheets/detail/ageing-and-health] (Accessed: 11/05/2020).

2. Pedersen A N \& Cederholm T (2014) Food Nutr Res, 58, 10.3402/fnr.v58.23364

3. Song X, Pérez-Cueto F, Bølling Laugesen S, van der Zanden L, Giacalone D (2019) Appetite 135, 10-19.

4. Addinsoft (2020) XLSTAT statistical and data analysis solution. 